\title{
LA RELACIÓN
} CORPORATIVO, LA ORIENTACIÓN AL APRENDIZAJE Y EL COMPROMISO ORGANIZACIONAL CON EL DESEMPEÑO ORGANIZACIONAL: UN ACERCAMIENTO TEÓRICO

\footnotetext{
Daniel Sierra Carpio Universidad Autónoma de Chihuahua

Recibido: 02 de septiembre de 2018 Aceptado: 24 de octubre de 2018
}

\section{RE}

D 1 present porta una revisión teórica que confirma la existencia de una relación significativa entre el emprendimiento corporativo, la orientación al aprendizaje, el compromiso organizacional y el desempeño organizacional. La revisión de literatura se desarrolló entre los meses de febrero de 2017 y mayo de 2018 en diferentes bases de datos y metabuscadores como EBSCO, Web of Science, Elsevier, Eme- rald, Redalyc y el buscador Google Scholar. Finalmente se propone un modelo a partir del cual se puede desarrollar una investigación que contribuya a la realización de futuros trabajos desde la perspectiva latinoamericana.

Palabras clave: Emprendimiento corporativo, orientación al aprendizaje, desempeño organizacional, compromiso organizacional. 


\section{Abstract}

This paper reports a theoretical review that confirms the existence of a significant relationship between corporate entrepreneurship, learning orientation, organizational commitment and organizational performance. The literature review was developed between february 2017 and may 2018 in different databases and metasearch engines such as EBSCO, Web of Science, Elsevier, Emerald, Redalyc and Google Scholar. Finally a model that contributes is proposed to the realization of futures research works from the perspective Latin American.

Keywords: Entrepreneurial orientation, learning orientation, firm performance, organizational commitment.

\section{INTRODUCCIÓN}

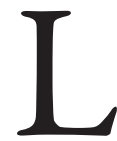

as orientaciones estratégicas, como lo son el Emprendimiento Corporativo (EC) y la Orientación al Aprendizaje (OA), se han considerado como antecedentes y potencializadores de diversas variables y que favorecen al aumento del Desempeño
Organizacional (DO) (Hakala, 2011). Otro constructo que ha reportado beneficio al desempeño organizacional es el Compromiso Organizacional (CO) (Mayer \& Schoorman, 1992). Sin embargo, se han desarrollado pocos estudios acerca de estos temas a nivel Latinoamérica. Una búsqueda en Scopus, llevada a cabo en el mes de mayo de 2018, arrojó datos al respecto: la mayor cantidad de estudios publicados acerca del EC son en Estados Unidos, con 332, mientras que el país latinoamericano con más publicaciones del tema es Brasil, con 25, seguido de México con 18. De manera similar, los estudios del OA los lidera Estados Unidos con 254, mientras que en la región latinoamericana, nuevamente Brasil se encuentra a la cabeza con cinco, seguido de México con cuatro. Finalmente, las investigaciones del $\mathrm{CO}$ eran en su mayoría realizadas por los Estados Unidos con 2281; en contra parte, en Latinoamérica, Brasil reportó 42 estudios, seguido de México con 20. Los datos anteriores hacen evidente la necesidad de aportaciones desde el ámbito latinoamericano para el desarrollo teórico de dichos constructos y sus implicaciones en la región.

Con base en lo anterior, el presente artículo tiene como objetivo desarrollar una investigación doctoral mediante la revisión de literatura con la intención de contestar la siguiente pregunta: ¿existen elementos teóricos que sugieran una relación existente entre el desempeño organizacional, el emprendimiento corporativo, la orientación al aprendizaje y el compromiso organizacional?

\section{REVISIÓN DE LITERATURA} Emprendimiento corporativo In los últimos años, los 4 estudios relacionados con el emprendimiento han cobrado mayor relevancia. Serrano-Bedia, PérezPérez, Palma-Ruiz \& LópezFernández (2016) realizaron una revisión sistemática de los números especiales de revistas enlistadas en el Índice de Citas de Ciencias Sociales - SSCI, por sus siglas en inglés Social Science Citation Index- con el fin de identificar los estudios que se publicaron acerca del emprendimiento en los años 2011 a 2013. Los autores identificaron tres tópicos que 
cobraban mayor relevancia: el emprendimiento social, el emprendimiento académico y el emprendimiento corporativo.

El emprendimiento corporativo se considera un atributo organizacional propagado a través de sus empleados con el fin de generar nuevas oportunidades de negocio, como por ejemplo, la creación de nuevas empresas o alianzas estratégicas, la renovación empresarial o la innovación de procesos o productos (Covin \& Miller, 2013; Covin \& Wales, 2011; Delgado Márquez, Luisa, \& Hurtado Torres, 2013; Lumpkin \& Dess, 1996; Sharma \& Chrisman, 1999).

Autores de los estudios del emprendimiento corporativo mencionan el término "new entry" (Covin \& Miller, 2013; Lumpkin \& Dess, 1996; Miller, 2011; Sharma \& Chrisman, 1999), que hace referencia al resultado que se da en la empresa después de llevar a cabo el proceso del emprendimiento. Sharma y Chrisman (1999) clasifican las new entries en tres tipos: 1) la creación de nuevos negocios dentro o fuera de una organización, 2) la transformación de negocios ya existentes por medio de la renovación estratégica y 3) la innovación dentro de la empresa, ya sea de procesos o productos. La figura 1 ilustra los tres tipos de new entries según los autores.

En la revisión de la literatura se distinguen dos maneras de abordar al emprendimiento corporativo para su estudio: (1) como un constructo formativo, compuesto por tres dimensiones llamadas innovación, toma de riesgos y proactividad (Covin \& Slevin, 1989; Miller, 2011); y (2) como un constructo reflectivo compuesto por cinco dimensiones independientes llamadas innovación, toma de riesgos, proactividad, autonomía y agresividad competitiva (Hughes \& Morgan, 2007; Lumpkin \& Dess, 1996). Para la medición de la visión de tres dimensiones se utiliza mayormente el cuestionario MCS - llamado así por las iniciales de los apellidos de los autores: Millers, Covin y Slevin(Covin \& Miller, 2013; Covin \& Wales, 2011) y para la visión de las cinco dimensiones independientes, los expertos (Covin \& Miller, 2013; Covin \& Wales, 2011) recomiendan usar el cuestionario creado por Hughes y Morgan (2007).

Compromiso organizacional 71 compromiso organi1 zacional es definido como una actitud, estado mental o psicológico de los empleados, el cual forma un vínculo con la organización (Llobet \& Fito, 2013; Meyer \& Allen, 1991). Los estudios de Meyer \& Allen (1984, 1991) clasifican al compromiso organizacional en tres tipos: (1) compromiso afectivo, (2) compromiso normativo y (3) de continuidad; esta clasificación fue efectuada después que los autores conciliaran y unificaran varios modelos y concepciones anteriores.

De acuerdo con Meyer \& Allen (1991), el compromiso afectivo es aquel en el que el empleado desea permanecer dentro de la organización debido a un apego emocional; el compromiso normativo se refiere al deseo de un empleado en seguir en la organización debido a un sentimiento de obligación, es decir, cuando el empleado siente que permanecer dentro de la empresa es lo "correcto" o es lo "moral" (Meyer \& Allen, 1984, 1991; 
Figura 1. Emprendimiento corporativo

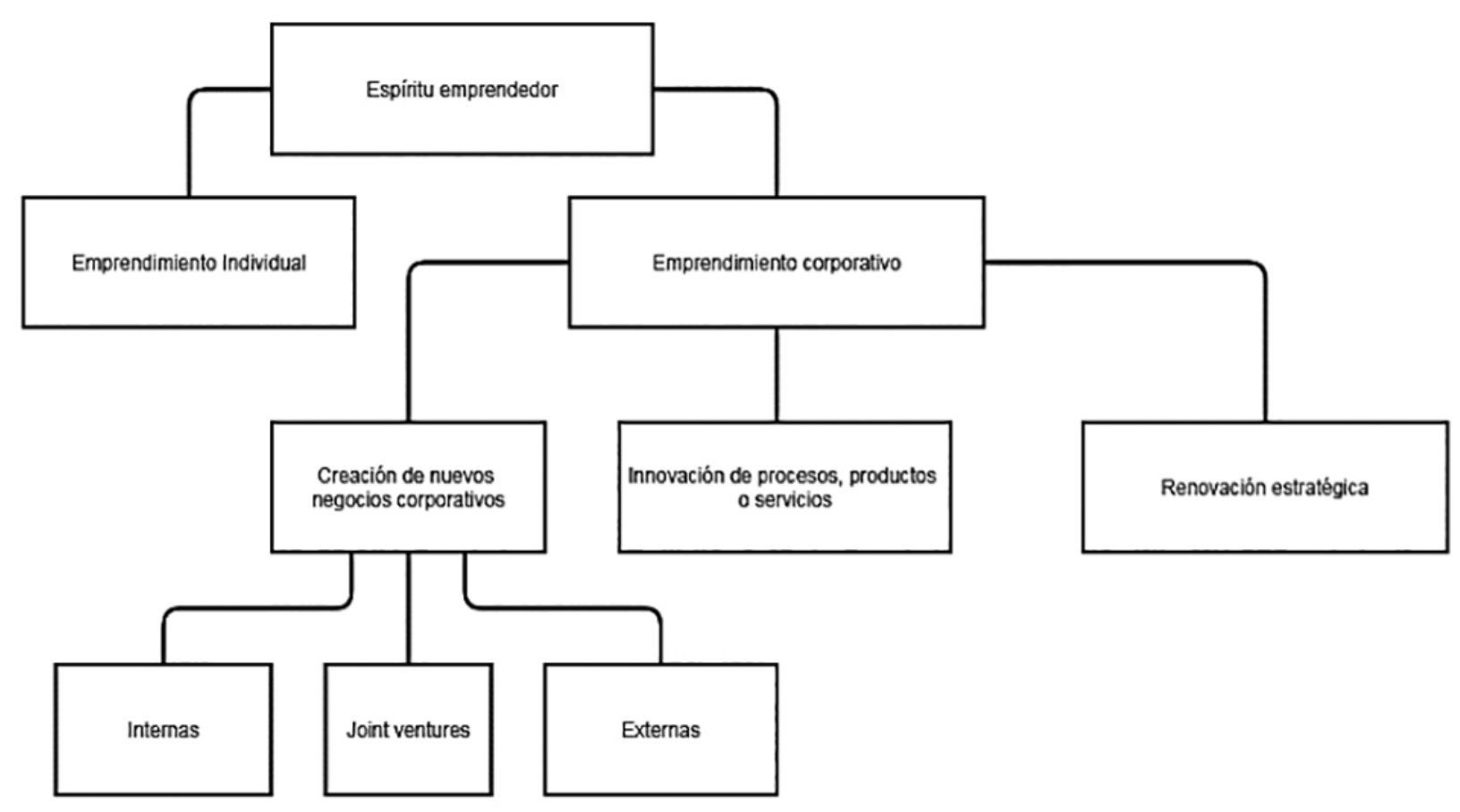

Fuente: Elaboración propia con base en Sharma \& Chrisman (1999)

Meyer \& Herscovitch, 2001). Finalmente, el compromiso de continuidad se refiere al hecho de permanecer en la organización debido al costo asociado a salir de ella (Meyer \& Allen, 1984; Meyer \& Herscovitch, 2001; Meyer, Stanley, Herscovitch, \& Topolnytsky, 2002).

\section{Orientación al aprendizaje}

T a orientación al aprendizaje es descrita por 1 Garrido \& Camarero
(2010) como una capacidad organizacional para generar mejor conocimiento y responder al ambiente en el que está inmersa, mejorando así la eficiencia. Esta capacidad organizacional se basa en conseguir conocimiento para generar nuevos valores y creencias organizacionales, modificar procesos, así como la forma en la que la organización aprende (Wang, 2008).
Mediante la revisión de literatura se encontró que la orientación al aprendizaje se mide como un constructo formativo compuesto por tres dimensiones conocidas como compromiso al aprendizaje, mentalidad abierta y visión compartida (Baker \& Sinkula, 1999).

El compromiso al aprendizaje indica que la organización está dispuesta a promover una cultura de aprendizaje. 
La mentalidad abierta, según Hakala (2011), está ligada al término "desaprender", pues es vital que una organización cuestione sus creencias, rutinas, rituales y procesos para que un verdadero aprendizaje ocurra. Finalmente, la visión compartida indica la dirección del aprendizaje, es decir, si en la organización prevalece un ambiente de ambigüedad, los empleados no sabrán qué aprender o en dónde aplicarlo. Esta dimensión es crucial para emprender nuevos proyectos (Garrido \& Camarero, 2010).

Desempeño organizacional

71 desempeño organi4 zacional es concebido como una métrica organizacional de eficacia y eficiencia que agrupa indicadores a niveles operativos y financieros, articulados sistemáticamente para satisfacer a los clientes y alcanzar las metas organizacionales en diferentes niveles (Del Castillo \& Vargas, 2009).

Los estudios consultados (Berger Werlang \& Follman Orth, 2017; Calantone, Cavusgil, \& Zhao, 2002; Carrizo Moreira \& Silva, 2013; Chavez, Yu, Jacobs, \& Feng,
2017; Covin \& Slevin, 1989; Garrido \& Camarero, 2010; Hughes \& Morgan, 2007; Linton \& Kask, 2017; Mayer \& Schoorman, 1992; Miller, 1983; Pratono \& Mahmood, 2015; Semrau, Ambos \& Kraus, 2016; Stopford \& Baden-Fuller, 1994; Vega-Vázquez, Cossío-Silva \& RevillaCamacho, 2016; Zahra, 1995; Zahra \& Covin, 1995; Zehir, Can \& Karaboga, 2015) arrojaron que para la medición del desempeño organizacional se utilizan indicadores financieros y no financieros, los cuales se pueden dividir en medidas objetivas y subjetivas. Las primeras son compuestas por indicadores financieros obtenidos directamente de los reportes de las propias empresas o consultoras financieras, mientras que las medidas subjetivas pueden utilizar tanto indicadores financieros como no financieros, pero desde la percepción de los encuestados, generalmente son obtenidos utilizando un instrumento con base en una escala Likert.

Entre los indicadores financieros se encuentran: la rentabilidad, el crecimiento de las ventas, el crecimiento de la compañía, el valor de las acciones, flujo de efectivo, rendimiento de los activos, entre otros (Shampy Kamboj, 2015), mientras que la satisfacción de cliente, la satisfacción de los empleados, la lealtad del cliente, el aumento de clientes, la reputación de la imagen, el desempeño de la marca y la experiencia son ejemplos de indicadores no financieros (Shampy Kamboj, 2015).

Según Vij \& Bedi (2012) los indicadores financieros son más difíciles de obtener, ya que los dueños o los gerentes no están dispuestos a compartir esa información, sin embargo, son más abiertos a compartir los indicadores no financieros.

\section{Metodología}

os estudios consultados
en la revisión de lite-
ratura se recuperaron en el periodo comprendido de febrero de 2017 y mayo de 2018. Se utilizaron las bases de datos EBSCO, Web of Science (ahora Clarivate Analytics), Elsevier, Emerald, Redalyc y el buscador Google Scholar. Las palabras clave utilizadas en las búsquedas incluyeron: emprendimiento corporativo, entrepreneurial orientation, entrepreneurship orientation, 
corporate entrepreneurship, learning orientation, compromiso organizacional, organizational commitment. Se contemplaron los artículos que incluían en su título o en sus objetivos planteados las palabras clave de la búsqueda; en cuanto a idioma de los artículos se seleccionaron aquellos que fueran en español, inglés o portugués.

\section{RESUlTADOS DE LA REVISIÓN DE LITERATURA}

Relación Emprendimiento Corporativo y Desempeño Organizacional

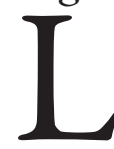

a mayoría de las investigaciones del emprendi-

miento corporativo se centran en estudiar el impacto de dicha variable en el desempeño organizacional y generalmente se ha reportado que el emprendimiento corporativo ejerce una influencia positiva sobre el desempeño organizacional (Chavez et al., 2017; Covin \& Wales, 2011; Hughes \& Morgan, 2007; Linton \& Kask, 2017; Lumpkin \& Dess, 2001; Miller, 2011; Pratono \& Mahmood, 2015; Semrau et al., 2016; Vega-Vázquez et al., 2016; Vij \& Bedi, 2012; Xu \& $\mathrm{Xu}, 2012$ ).

En algunos estudios se reporta que el emprendimiento corporativo es un factor nulo o inclusive de decremento para el desempeño organizacional, debido a factores como la edad de la empresa, el grado de turbulencia ambiental al que se enfrenta la organización (Pratono \& Mahmood, 2015), algunas estructuras formales de la empresa (Hakala, Sirén \& Wincent, 2016), ciertos aspectos de la cultura del país (Bachmann, Engelen \& Schwens, 2016; Semrau et al., 2016) o el grado del involucramiento de la familia en los negocios familiares (Bauweraerts \& Colot, 2017; Revilla, PérezLuño \& Nieto, 2016).

\section{Relación Emprendimiento} Corporativo, Orientación al Aprendizaje y Desempeno Organizacional

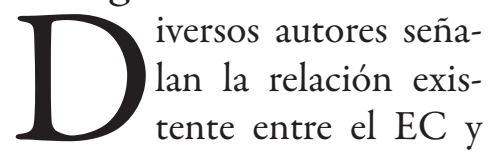
la OA (Daryani \& Karimi, 2018; Spillecke \& Brettel, 2014; Wang, 2008); en sus estudios llegan a conclusiones similares, en la que la orien- tación al aprendizaje modera la relación existente entre el emprendimiento corporativo y el desempeño organizacional. La diferencia entre estos estudios es que para Daryani \& Karimi (2018) el EC ejerce una infuencia sobre la OA, mientras que para Spillecke \& Brettel (2014) y Wang (2008), la relación es inversa, es decir, la OA ejerce una influencia positiva sobre el EC, al menos de manera indirecta (Spillecke \& Brettel, 2014), sin embargo, todos los autores concluyen que la presencia del EC junto con la OA mejorará el desempeño organizacional.

Otros autores encontraron una relación positiva entre la $\mathrm{OA}$, la innovación y el desempeño organizacional (Berger \& Follman, 2017; Calantone et al., 2002; Garrido \& Camarero, 2010). Los resultados sugieren que la orientación al aprendizaje tiene una relación positiva con la innovación y estos a su vez mejoran el desempeño organizacional; cabe resaltar que la innovación es una dimensión del EC (Covin \& Slevin, 1989; Lumpkin \& Dess, 1996). 
Relación entre el Emprendimiento corporativo y el Compromiso organizacional

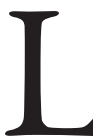

a revisión de literatura también reportó una relación existente en el EC y el CO (De Clercq \& Belausteguigoitia, 2007; De Clercq, Dimov \& Thongpapanl, 2010; Farrukh, Chong, Mansori \& Ravan, 2017). Las investigaciones realizadas por De Clercq et al. (2010) y por Farrukh et al. (2017) destacan que el compromiso organizacional ejerce una influencia positiva en el emprendimiento corporativo, sobre todo con el llamado compromiso afectivo.

Por otro lado, De Clercq \& Belaisteguigoitia (2007), reportan también una relación positiva entre los constructos mencionados, sin embargo, sus resultados sugieren que es el emprendimiento corporativo el que favorece al compromiso organizacional y no al revés.

Es de destacar que todos los estudios mencionados en esta sección midieron el compromiso organizacional con lo establecido por Meyer \& Allen (1991).
Relación entre el Compromiso organizacional y el Desempeño organizacional 7 iversos estudios han relacionado el compromiso organizacional con diversas manifestaciones del desempeño dentro de la empresa, por ejemplo el desempeño laboral (Meyer, Becker \& Vandenberghe, 2004; Randall, 1988) o el desempeño de los equipos (Wombacher \& Felfe, 2017). De manera similar, autores de distintas revisiones de literatura y meta-análisis registran estudios donde se pone de manifiesto esta relación entre compromiso organizacional y distintos tipos de desempeño, incluido el organizacional (Mayer \& Schoorman, 1998; Meyer et al., 2002; Steinhaus \& Perry, 1996; Yahaya \& Ebrahim, 2016).

Entre los estudios que relacionan el DO y el CO está el llevado a cabo por Meyer \& Schoorman (1992), en donde los resultados sugieren una relación positiva entre el compromiso de valor (equivalente al compromiso afectivo) que influye positivamente al desempeño organizacional. De igual manera, Carrizo \& Sil- va (2013), así como Balfour \& Wechsler (1991), destacan que las bases de un buen desempeño organizacional se encuentran en el compromiso organizacional.

\section{Conclusiones}

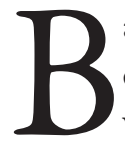
asados en la información obtenida en la revisión de literatura, se puede establecer que sí existen elementos teóricos que manifiesten una relación entre el emprendimiento corporativo, la orientación al aprendizaje, el compromiso organizacional y el desempeño organizacional; además, la teoría sugiere una relación positiva y también que al combinarse dos o más, el desempeño organizacional se ve beneficiado.

Por tanto, es factible establecer que existen las siguientes relaciones:

- El Compromiso Organizacional ejerce una relación positiva y significativa con el Emprendimiento Corporativo.

- El Compromiso Organizacional ejerce una relación positiva y significativa con el Desempeño Organizacional. 
- El Compromiso Organizacional ejerce una relación positiva y significativa con la Orientación al Aprendizaje

- El Emprendimiento Corporativo ejerce una relación positiva y significativa sobre el Desempeño Organizacional

- La Orientación al Aprendizaje ejerce una relación po- sitiva y significativa sobre el Emprendimiento Corporativo.

- La Orientación al Aprendizaje ejerce una relación positiva y significativa en el desempeño organizacional

Dichas relaciones pueden ponerse a prueba para aceptarse o rechazarse en una futura investigación en la que los resultados contribuyan a engrosar los estudios de estas variables en Latinoamérica y ayuden a las organizaciones mexicanas al aprovechamiento de sus recursos para la mejora de su desempeño.

\section{REFERENCIAS}

Bachmann, J. T., Engelen, A., \& Schwens, C. (2016). Toward a Better Understanding of the Association Between Strategic Planning and Entrepreneurial Orientation - The Moderating Role of National Culture. Journal of International Management, 22(4), 297-315. https://doi.org/10.1016/j.intman.2016.03.002

Baker, W. E., \& Sinkula, J. M. (1999). The Synergistic Effect of Market Orientation and Learning Orientation on Organizational Performance. Journal of the Academy of Marketing Science, 27(4), 411-427.

Balfour, D. L., \& Wechsler, B. (1991). Commitment and Performance, in Public Organizations. Public Productivity \& Management Review, 14(4), 355-367. Retrieved from http://www.jstor.org/stable/3380952

Bauweraerts, J., \& Colot, O. (2017). Exploring nonlinear effects of family involvement in the board on entrepreneurial orientation. Journal of Business Research, 70, 185-192. https://doi.org/10.1016/j.jbusres.2016.08.020

Berger Werlang, N., \& Follman Orth, D. (2017). Orientação para aprendizagem, inovatividade organizacional e desempenho organizacional sob a ótica de empresas internacionalizadas. Revista GEINTEC, 7(3), 3923-3938. https://doi.org/10.7198/geintec. v7.i3.866

Calantone, R. J., Cavusgil, V., \& Zhao, Y. (2002). Learning orientation, firm innovation capability, and firm performance. Industrial Marketing Management, 31(6), 515-524. 
Carrizo Moreira, A., \& Silva, P. M. (2013). Market Orientation, Innovation and Organizational Commiment in Industrial firms. Tržište, (2), 123-142.

Chávez, R., Yu, W., Jacobs, M. A., \& Feng, M. (2017). Manufacturing capability and organizational performance: The role of entrepreneurial orientation. International Journal of Production Economics, 184(March 2016), 33-46. https://doi.org/10.1016/j. ijpe.2016.10.028

Covin, J. G., \& Miller, D. (2013). International Entrepreneurial Orientation: Conceptual considerations, research themes, measurement issues, and future research direction. Entrepreneurship: Theory and Practice, 38(1), 11-44. Retrieved from doi: 10.1111/j.15406520.2010.00432.x

Covin, J. G., \& Slevin, D. P. (1989). Strategic management of small firms in hostile and benign enviroments. Strategic Management Journal, 10(1), 75-87. https://doi. org/10.1002/smj.4250100107

Covin, J. G., \& Wales, W. J. (2011). The Measurement of entrepreneurial orientation. Entrepreneurship: Theory and Practice, 36(4), 677-702. Retrieved from 10.1111/j.15406520.2010.00432.x

Daryani, M. A., \& Karimi, A. (2018). Effect of Corporate Entrepreneurship on Firm Perfor mance in Iranian ASMEs: The Mediation Role of Knowledge Crea tion and Learning Orientation. Journal of Agricultural Science and Technology, 19, 261-277. Retrieved from https://sci-hub.tw/http://ir.jkuat.ac.ke/handle/123456789/3674

De Clercq, D., \& Belausteguigoitia Rius, I. (2007). Organizational commitment in Mexican small and medium-sized firms: The role of work status, organizational climate, and entrepreneurial orientation. Journal of Small Business Management, 45(4), 467-490. https://doi.org/10.1111/j.1540-627X.2007.00223.x

De Clercq, D., Dimov, D., \& Thongpapanl, N. (Tek). (2010). The moderating impact of internal social exchange processes on the entrepreneurial orientation-performance relationship. Journal of Business Venturing, 25(1), 87-103. https://doi.org/10.1016/j.jbusvent.2009.01.004

Del Castillo, C., \& Vargas, B. (2009). el proceso de gestión y el desempeño organizacional Una aproximación a la nueva gestión pública desde el ámbito de los gobiernos locales. Journal of Economics, Finance and Administrative Science, 14(26), 57-80. Retrieved from http://www.redalyc.org/pdf/3607/360733606003.pdf

Delgado Márquez, B. L., Luisa, D. M., \& Hurtado Torres, N. E. (2013). Emprendimiento corporativo y capital social: Un análisis de empresas intensivas en tecnología. Revista Venezolana de Gerencia, 18(61), 62-85.

Farrukh, M., Chong, W. Y., Mansori, S., \& Ravan Ramzani, S. (2017). Intrapreneurial behaviour: the role of organizational commitment. World Journal of Entrepreneurship, 
Management and Sustainable Development, 13(3), 243-256. https://doi.org/10.1108/ WJEMSD-03-2017-0016

Garrido, M. J., \& Camarero, C. (2010). Assessing the impact of organizational learning and innovation on performance in cultural organizations. International Journal of Nonprofit and Voluntary Sector Marketing, 232(September 2009), 215-232. https://doi. org/10.1002/nvsm

Hakala, H. (2011). Strategic Orientations in Management Literature: Three Approaches to Understanding the Interaction between Market, Technology, Entrepreneurial and International Journal of Management Reviews, 13, 199-217. https://doi.org/10.1111/ j.1468-2370.2010.00292.x

Hakala, H., Sirén, C., \& Wincent, J. (2016). Entrepreneurial Orientation and International New Entry: The Moderating Role of Autonomy and Structures in Subsidiaries. Journal of Small Business Management, 54, 90-112. https://doi.org/10.1111/jsbm.12294

Hughes, M., \& Morgan, R. E. (2007). Deconstructing the relationship between entrepreneurial orientation and business performance at the embryonic stage of firm growth. Industrial Marketing Management, 36(5), 651-661. https://doi.org/10.1016/j.indmarman.2006.04.003

Linton, G., \& Kask, J. (2017). Configurations of entrepreneurial orientation and competitive strategy for high performance. Journal of Business Research, 70, 168-176. https://doi. org/10.1016/j.jbusres.2016.08.022

Llobet, J., \& Fito, M. A. (2013). Organizational Commitment, job satisfaction and intention to stay: Literature review. Intangible Capital, 9(4), 1068-1079. https://doi.org/10.3926/ ic. 475

Lumpkin, G. T., \& Dess, G. G. (1996). Clarifying the Entrepreneurial Orientation Construct and Linking It to Performance Author. The Academy of Management Review, 21(1), 135-172. Retrieved from http://www.jstor.org/stable/258632

---- (2001). Linking two dimensions of entrepreneurial orientation to firm performance: The moderating role of environment and industry life cycle. Journal of Business Venturing, 16(5), 429-451. https://doi.org/10.1016/S0883-9026(00)00048-3

Mayer, R. C., \& Schoorman, F. D. (1992). Predicting Participation and Production Outcomes through a Two-Dimensional Model of Organizational Commitment. The Academy of Management Journal, 35(3), 671-684. Retrieved from http://www.jstor.org/stable/256492

Mayer, R. C., \& Schoorman, F. D. (1998). Differentiating antecedents of organizational commitment: a test of March and Simon's model. Journal of Organizational Behavior, 19(April 1996), 15-28. https://doi. 
Miller, D. (2011). Miller (1983) Revisited: A reflection on EO research and some suggestions for the future. Entrepreneurship: Theory and Practice, 35(5), 873-894. Retrieved from 10.1111/j.1540-6520.2011.00457.x

Pratono, A. H., \& Mahmood, R. (2015). Entrepreneurial orientation and firm performance: How can micro, small and medium-sized enterprises survive environmental turbulence? Pacific Science Review B: Humanities and Social Sciences, 1(2), 85-91. https:// doi.org/10.1016/j.psrb.2016.05.003

Randall, D. M. (1988). Multiple Roles and Organizational Commitment. Journal of Organizational Behavior, 9(4), 309-317. Retrieved from http://www.jstor.org/stable/2488089

Revilla, A. J., Pérez-Luño, A., \& Nieto, M. J. (2016). Does Family Involvement in Management Reduce the Risk of Business Failure? The Moderating Role of Entrepreneurial Orientation. Family Business Review, 29(4), 365-379. https://doi org/10.1177/0894486516671075

Semrau, T., Ambos, T., \& Kraus, S. (2016). Entrepreneurial orientation and SME performance across societal cultures: An international study. Journal of Business Research, 69(5), 1928-1932. https://doi.org/10.1016/j.jbusres.2015.10.082

Serrano-Bedia, A. M., Pérez-Pérez, M., Palma-Ruiz, M., \& López-Fernández, M. C. (2016). Emprendimiento: visión actual como disciplina de investigación. Un análisis de los números especiales publicados durante 2011-2013. Estudios Gerenciales, 32(138), 82-95.

Shampy Kamboj, Z. R. (2015). Marketing capabilities and firm performance: literature review and future research agendat. International Journal of Productivity and Performance Management, 64(4), 544-566. Sharma, P., \& Chrisman, J. J. (1999). Toward a Reconciliation of the Definitional Issues in the Field of Corporate Entrepreneurship. Entrepreneurship: Theory and Practice, 33, 273-304.

Spillecke, S. B., \& Brettel, M. (2014). Antecedents and Performance Implications of a Sales Department's Learning and Entrepreneurial Orientation in SMEs. Journal of Small Business Management, 52(3), 407-426.

Steinhaus, C. S., \& Perry, J. L. (1996). Organizational Commitment : Does Sector Matter ? L. Pulic Productivity \& Management Review, 19(3), 278-288. Retrieved from http:// www.jstor.org/stable/3380575

Stopford, J. M., \& Baden-Fuller, C. W. F. (1994). Creating corporate entrepreneurship. Strategic Management Journal, 15(7), 521-536. https://doi.org/10.1002/smj.4250150703

Vega-Vázquez, M., Cossío-Silva, F. J., \& Revilla-Camacho, M. Á. (2016). Entrepreneurial orientation-hotel performance: Has market orientation anything to say? Journal of Business Research, 69(11), 5089-5094. https://doi.org/10.1016/j.jbusres.2016.04.085 
Vij, S., \& Bedi, H. S. (2012). Relationship Between Entrepreneurial Orientation and Business Performance: A Review of Literature. IUP Journal of Business Strategy, 9(3), 17-31. Retrieved from http://search.ebscohost.com/login.aspx ?direct $=$ true $\& \mathrm{db}=$ buh $\& A N=$ $83439047 \&$ site $=$ ehost-live

Wang, C. L. (2008). Entrepreneurial orientation, learning orientation and firm performance. Entrepreneurship: Theory and Practice, 44(0), 635-657.

Wombacher, J. C., \& Felfe, J. (2017). Dual commitment in the organization: Effects of the interplay of team and organizational commitment on employee citizenship behavior, efficacy beliefs, and turnover intentions. Journal of Vocational Behavior, 102(May), 1-14. https://doi.org/10.1016/j.jvb.2017.05.004

$\mathrm{Xu}, \mathrm{T} ., \& \mathrm{Xu}, \mathrm{Y} .(2012)$. A literature review of relationship between entrepreneurial orientation and firm performance. 2012 International Symposium on Management of Technology (ISMOT), 128-131. https://doi.org/10.1109/ISMOT.2012.6679442

Yahaya, R., \& Ebrahim, F. (2016). Leadership styles and organizational commitment: literature review. Journal of Management Development, 35(2), 190-216. https://doi. org/10.1108/JMD-01-2015-0004

Zahra, S. A. (1995). Corporate entrepreneurship and financial performance: The case of management leveraged buyouts. Journal of Business Venturing, 10(3), 225-247. https:// doi.org/10.1016/0883-9026(94)00024-O

Zahra, S. A., \& Covin, J. G. (1995). Contextual influences on the corporate entrepreneurship-performance relationship: A longitudinal analysis. Journal of Business Venturing, 10(1), 43-58. https://doi.org/10.1016/0883-9026(94)00004-E

Zehir, C., Can, E., \& Karaboga, T. (2015). Linking Entrepreneurial Orientation to Firm Performance: The Role of Differentiation Strategy and Innovation Performance. Procedia-Social and Behavioral Sciences, 210, 358-367. https://doi.org/10.1016/j.sbspro.2015.11.381 